\title{
Editorial: Get serious
}

\author{
Mark Winands \\ Department of Data Science and Knowledge Engineering, Maastricht University, The Netherlands \\ E-mail:m.winands@maastrichtuniversity.nl
}

Games can have multiple purposes. They are not only a source of entertainment, but they can also provide an intellectual challenge. At the moment games have an educational or training aspect, they are called serious games. These games are being applied for instance in the health sector, business, or security. There is a link between the AI research applied in serious games and the type of research usually being discussed in the ICGA journal. For example, Monte-Carlo Tree Search, which is the default search technique for Go, has been used to control the behavior of virtual characters in a simulated surgery room environment for medical staff training (Sanselone et al., 2014). The literature indicates that serious games can be an effective teaching tool, but is fragmented when it comes to the factors that shape the users' experience and views. In Let players evaluate serious games. Design and validation of the Serious Games Evaluation Scale, Emmanuel Fokides, Penelope Atsikpasi, Polyxeni Kaimara and Ioannis Deliyannis provide a tool that simultaneously assesses many factors that shape the users' views when playing serious games.

The research how games may have been played throughout history, and how they influenced and transferred between different civilizations is a serious business as well. This issue contains a report summarizing a recent Dagstuhl research meeting, in which the foundations of the new research field of Digital Archæoludology has been established. Research in this field aims to use techniques from the field of AI, and other computational and data-driven techniques, to study traditional games.

This issue also reflects on the achievements of the computer chess engines participating in TCEC 14, TCEC 15, and TCEC Cup 3. Finally, I am happy to report that we have a call for papers on a special issue on Combinatorial Games. It is projected to be published in the ICGA journal for 2020. Jos Uiterwijk and Richard Nowakowski will be responsible for this special issue.

Mark Winands

\section{REFERENCES}

Sanselone, M., Sanchez, S., Sanza, C., Panzoli, D. \& Duthen, Y. (2014). Constrained control of nonplaying characters using Monte Carlo tree search. In 2014 IEEE Conference on Computational Intelligence and Games (pp. 208-215). doi:10.1109/CIG.2014.6932886. 